
\title{
28 Research Square \\ Functional Connectivity in Parkinson's Disease Patients with Mild Cognitive Impairment
}

\section{Qingguang Wang}

the Affiliated Jiangyin People's Hospital of Southeast University Medical College

Wei He

the Affiliated Jiangyin People's Hospital of Southeast University Medical College

\section{Dinghua Liu}

the Affiliated Jiangyin People's Hospital of Southeast University Medical College

\section{Bojun Han}

the Affiliated Jiangyin People's Hospital of Southeast University Medical College

\section{Qitao Jiang}

the Affiliated Jiangyin People's Hospital of Southeast University Medical College

Jiali Niu ( $\square$ jialiniu@yahoo.cn )

Jingjiang People's hospital https://orcid.org/0000-0002-4993-2488

\section{Yunlong Ding}

Jingjiang People's Hospital

\section{Research article}

Keywords: Parkinson's disease, Mild cognitive impairment, Resting-state functional magnetic resonance imaging, Functional connectivity

Posted Date: April 8th, 2020

DOI: https://doi.org/10.21203/rs.3.rs-20315/v1

License: (c) (1) This work is licensed under a Creative Commons Attribution 4.0 International License.

Read Full License

Version of Record: A version of this preprint was published at International Journal of General Medicine on June 1st, 2021. See the published version at https://doi.org/10.2147/IJGM.S300422. 


\section{Abstract}

Background: To explore the alteration of pattens of anatomical and functional connectivity (FC) of posterior cingulate cortex (PCC) in Parkinson's disease (PD) patients with cognitive dysfunction and the relationship between the connection strengths and cognitive state $\mathbb{Z}$

Methods: We prospectively enrolled 20 PD patients with mild cognitive impairment (PD-MCI), 13 PD patients with normal cognition (PD-NC) and 13 healthy controls (HCs). All subjects underwent clinical evaluations and MRI scans. By collecting, preprocessing and FC analyzing resting-state functional magnetic resonance imaging (rs-fMRI) data, we extracted default mode network (DMN) patterns, compared the differences in DMN between the three groups and the analyzed the correlation between FC value with the commonly used neuropsychological testing.

Results: There were not significant differences with regard to demographic data among the three groups. The PD-MCl showed significant worse performances in general cognition, and PD-NC and HCs showed comparable performances of cognitive function. Cognitive-related differences in DMN were detected in the bilateral precuneus (BPcu). Compared with the HCs, PD-NC and PD-MCl showed significantly decreased FC within BPcu (both $\mathrm{P}<0.001)$. For PD-MCl, the rho of the the Fisher's Z-transformed FC ( $\mathrm{zFC})$ value within BPcu with the TMTA, DSST and CFT-20min were $-0.50,0.66$ and 0.47 , respectively. For PD$\mathrm{NC}$, the rho of the zFC value within BPcu with the MMSE was 0.58.

Conclusions: Our research found that BPcu was the cognitive related region in DMN. As cognition declines, FC within BPcu weaken. For PD-MCl, the higher the FC values within BPcu were related to the better the performances of TMTA, DSST and CFT-20 min DR. For PD patients with normal cognition, the FC within BPcu were positively correlated with scores of MMSE.

\section{Background}

Parkinson's disease (PD) is a chronic and progressive neurodegenerative disorder, decreasing the quality of life and placing serious social and economic burdens[1]. In China, the prevalence of PD is 1700 per 100,000 in individuals older than 65 years, which is similar to the rate reported in developed countries[2, 3]. PD is usually characterized by motor symptoms that the main clinical manifestations are bradykinesia, rest tremor present $[$ muscular rigidity and postural instability[4],but may lead to mild cognitive impairment and dementiaロEvidence implicates that patients with PD are six times more likely to develop dementia than healthy people[5]. In order to investigate the cognitive related changes of gray matter structure and function in PD patients, this study using resting state functional magnetic resonance imaging (rs-fMRI) to assess the pattens of anatomical and functional connectivity (FC) damage in PD patients with normal cognition (PD-NC) and PD patients with mild cognitive impairment (PD-MCl). Furthermore【we analysis the relationship between the connection strengths and cognitive state[

\section{Methods}




\subsection{Participants}

20 PD-MCl, 13 PD-NC and 13 healthy controls (HCs) were employed in the present study through newspaper advertisements. All subjects underwent clinical evaluations and MRI scans. One PD-MCl and one PD-NC were excluded from the imaging analysis because of the excessive motion artifacts. The study was approved by the Research Ethics Committee of the Affiliated Jiangyin People's Hospital of Southeast University Medical College and written informed consent was obtained from all participants.

\subsection{Clinical Evaluation}

Each subject underwent the comprehensive clinical evaluations, including the demographic information, history of past illness and neuropsychological testing. Mini-Mental State Examination (MMSE) were used to assess the general cognition. The neuropsychological battery mainly comprised of Auditory Verbal Learning Test-20-min delayed recall (AVLT-20 min DR), the Rey-Osterrieth Complex Figure Test (CFT20 min DR), Trail Making Test (TMT)-A and B, Verbal Fluency Test (VFT), Digital Symbol Substitution Test (DSST) and Clock Drawing Test (CDT) was used to evaluate the episodic memory, visuospatial function, information processing speed and executive function.

\subsection{Inclusion and exclusion criteria}

The diagnosis of PD was made following the 2016 Chinese diagnostic criteria for PD[6].

The diagnosis of $\mathrm{MCl}$ was made following the recommendations of Petersen et al.[7] and others[8, 9]: (a) subjective memory impairment corroborated by the subject and an informant; (b) objective memory performance documented by an AVLT-20 min DR score less than or equal to 1.5 SD of age- and education-adjusted norms (cut-off of $\leq 4$ correct responses on 12 items for patients with $\geq 8$ years of education); (c) MMSE score of 24 or higher; (d) Clinical Dementia Rating (CDR) of 0.5; (e) no or minimal impairment in activities of daily living; $(f)$ absence of dementia or insufficient dementia to meet the National Institute of Neurological and Communicative Disorders and Stroke and the Alzheimer's Disease and Related Disorders Association (NINCDS-ADRDA) Alzheimer's criteria. In addition, the controls were required to have a CDR of 0 , an MMSE score $\geq 26$, and an AVLT-20 min DR score $\geq 4$ for subjects with 8 or more years of education.

Participants were excluded from the study if they had a history of known stroke, alcoholism, head injury, Parkinson's disease, epilepsy, major depression or other neurological or psychiatric illness, major medical illness, or severe visual or hearing loss.

\subsection{MRI data acquisition}

The subjects were scanned using a General Electric 1.5 T scanner (General Electric Medical Systems, USA) with a homogeneous birdcage head coil. Subjects lay supine with the head snugly fixed by a belt and foam pads to minimize head motion. Conventional axial fast relaxation fast spin echo sequence T2 weighted anatomic MR images were obtained to rule out major white matter changes, cerebral infarction or other lesions: repetition time $(T R)=3500$ ms; echo time $(T E)=103$ ms; flip angle $(F A)=90$; acquisition 
matrix = 3209 192; fieldof view $(F O V)=2409240$ mm; thickness = 6.0 mm; gap = 0 mm; no. of excitations $(\mathrm{NEX})=2$.0. High-resolution T1-weighted axial images covering the whole brain were acquired using a 3D spoiled gradient echo sequence as follow: TR = $9.9 \mathrm{~ms} ; \mathrm{TE}=2.1 \mathrm{~ms} ; \mathrm{FA}=150$; acquisition matrix = 2569 192; FOV = 240 mm 9240 mm; thickness = $2.0 \mathrm{~mm}$; gap = 0 mm. The functional scans (T2* weighted images) involved the acquisition of 30 contiguous axial slices using a GRE-EPI pulse sequence: $\mathrm{TR}=3,000$ ms; TE = 40 ms; FA= 90; acquisition matrix = 649 64; FOV = 2409240 mm; thickness $=4.0 \mathrm{~mm}$; gap $=0 \mathrm{~mm}$ and $3.7593 .75 \mathrm{~mm} 2$ inplane resolution parallel to the anterior commissure-posterior commissure line. This acquisition sequence generated 142 volumes in 7 min and 6 s. All subjects have eyes closed during scanning.

\subsection{MRI data preprocessing}

The preprocessing was performed by using Data Processing Assistant for Resting-State fMRI (DPARSF) (Yan \& Zang, 2010, http://www.restfmri.net), which is based on Statistical Parametric Mapping (SPM8) (http://www.fil.ion.ucl.ac.uk/spm) and Resting-State fMRI Data Analysis Toolkit (REST, Song et al., 2011. http://www.restfmri.net). Data analyses of groups were conducted with SPM5 toolkit (http://www.fil.ion.ucl.ac.uk/spm). The first eight volumes of the scanning session were discarded to allow for T1 equilibration effects. The remaining images were corrected for timing differences and motion effects. No translation or rotation parameters of head motion in any given data set exceeded \pm $3 \mathrm{~mm}$ or \pm 3 . The resulting images were spatially normalised into the SPM5 Montreal Neurological Institute echo-planar imaging template using the default settings and resampling to $3 \times 3 \times 3 \mathrm{~mm}^{3}$ voxels, and smoothed with a Gaussian kernel of $6 \times 6 \times 6 \mathrm{~mm}$. Then, the linear trend of time courses was removed and the resulting $f M R I$ data were band-pass filtered $(0.01<f<0.08 \mathrm{~Hz})$.

\subsection{FC analysis}

The posterior cingulate cortex (PCC) hub [coordinate in the MNI space: $-2,-45,34$ ] was selected to generate a 6-mm radius spherical seed region[10, 11], followed by coregistration to the functional data. Individual time courses were extracted based on the coregistered seed region. Furthermore, voxel-wise cross-correlation (CC) values between the seed region and the whole brain were calculated. A Fisher's Ztransformation was then applied to improve the normality of the $\mathrm{CC}$ values. Finally, the individual default mode network (DMN) patterns were obtained. Additionally, the mean time series of global, white matter, cerebrospinal fluid signals and head motion parameters were introduced as covariates of no interest.

\subsection{Voxelwise-based grey matter volume correction}

To explore the FC changes that cannot be attributed to anatomical difference, the voxel's likelihood of containing grey matter were introduced as nuisance variables. Firstly, voxel-based morphometry (VBM) was used to explore grey matter volume maps of every subject. These maps were transformed into the same standard space as the rs-fMRI images using affine linear registration. Further, the resampled data were smoothed as the corresponding functional data $(8 \mathrm{~mm})$. Finally, the resulting voxelwise grey matter volume maps were input as covariates in the analysis of functional data. The voxelwise-based grey matter volume correction was used for each subject[12]. 


\subsection{Statistical analysis}

\subsubsection{Demographic and Neuropsychological Data}

Kruskal Wallis tests followed by Dunn-Bonferroni post hoc were performed as the neuropsychological data were not normally distributed ( $P<0.05$ for Kolmogorov-Smirnov test). The $\chi 2$ tests were performed for gender. The SPSS 22.0 software was used and the statistical significance was set at $P<0.05$.

\subsubsection{Group-level analyses of the DMN}

Within groups: to determine the patterns of DMN in each of four groups, the spatial maps of DMN IC in each group were submitted to a random-effect analysis using one-sample t-tests. The thresholds were set at a corrected $P<0.01$, determined by Monte Carlo simulation for multiple comparison (single voxel $P$ value $=0.01$, a minimum cluster size of $6156 \mathrm{~mm} 3, \mathrm{FWHM}=6 \mathrm{~mm}$;

http://afni.nimh.nih.gov/pub/dist/doc/ manual/AlphaSim.pdf).

Between groups: One-way analysis of variance (ANOVA) was performed to explore the different DMN patterns among HCs, PD-NC and PD-MCl. The effects of age, gender and education were corrected[13, 14]. The statistical thresholds were set at an AlphaSim-corrected $P<0.01$ as determined by Monte Carlo simulation (single voxel $P$ value $=0.01$, a minimum cluster size of $6156 \mathrm{~mm} 3, \mathrm{FWHM}=6 \mathrm{~mm}$; http://afni.nimh.nih.gov/pub/dist/doc/manual/ AlphaSim.pdf). The regions survived the Monte Carlo simulations were taken as ROls, and the Fisher's Z-transformed FC $(\mathrm{zFC})$ strengths for each subject were extracted for further analysis.

\subsubsection{Characteristic of disease-related difference in DMN}

Post hoc tests were performed to explore the different DMN patterns of regions with disease-related changes among three groups. ANOVA analyses followed by the least-significant difference (LSD) analyses were utilized. To explore the cognitive significances of the disease-related changes in DMN, the spearman correlation analyses were performed between the zFC strengths and the neuropsychological performances. The SPSS 22.0 software was used and the statistical significance was set at $P<0.05$.

\section{Results}

\subsection{Demographic and Cognitive Data}

As shown in Table 1, the PD-MCl showed significant worse performances in general cognition (i.e. MMSE), episodic memory (i.e. AVLT-20 min DR and CFT-20 min DR), visuospatial function (i.e. CDT), information processing speed (i.e. DSST and TMT-A) and executive function (i.e. TMT-B and VFT, all $P<$ 0.05). Furthermore, the PD-NC and HCs showed comparable performances of cognitive function (all $P>$ $0.05)$. It should be noted that there were not significant differences with regard to demographic data among the three groups (all $P>0.05$ ). 
Table 1

Demographic and neuropsychological data for HCs, PD with normal cognition and PD with $\mathrm{MCl}$

\begin{tabular}{|c|c|c|c|c|}
\hline & $\begin{array}{l}\text { PD with MCl } \\
(n=20)\end{array}$ & $\begin{array}{l}\text { PD with normal cognition } \\
(n=13)\end{array}$ & $\begin{array}{l}\mathrm{HC} \\
(n=13)\end{array}$ & $\begin{array}{l}\mathrm{P} \\
(\mathrm{KW})\end{array}$ \\
\hline Age (years) & $72.6 \pm 6.1$ & $70.4 \pm 6.4$ & $70.2 \pm 7.1$ & 0.233 \\
\hline Gender (M/F) & $12 / 8$ & $7 / 6$ & $4 / 9$ & 0.247 \\
\hline Education (years) & $11.7 \pm 3.3$ & $12.2 \pm 3.9$ & $12.1 \pm 3.5$ & 0.688 \\
\hline MMSE & $26.9 \pm 3.1^{\star \star \star}$ & $28.5 \pm 1.5$ & $28.8 \pm 1.4$ & $<0.001$ \\
\hline AVLT-20 min DR & $2.9 \pm 1.2^{\star \star \star}$ & $6.9 \pm 1.1$ & $7.7 \pm 1.5$ & $<0.001$ \\
\hline CFT-20 min DR & $13.3 \pm 4.9^{\star \star \star}$ & $17.5 \pm 4.5$ & $19.2 \pm 4.9$ & $<0.001$ \\
\hline CDT & $8.0 \pm 2.1^{*}$ & $9.2 \pm 1.4$ & $8.9 \pm 1.0$ & 0.037 \\
\hline DSST & $33.8 \pm 7.8^{\star \star \star}$ & $41.2 \pm 8.8$ & $40.3 \pm 10.7$ & $<0.001$ \\
\hline TMT-A (second) & $75.8 \pm 14.5^{\star}$ & $63.6 \pm 19.8$ & $66.2 \pm 20.1$ & 0.019 \\
\hline VFT & $17.1 \pm 4.1^{\star \star \star}$ & $19.9 \pm 4.4$ & $21.1 \pm 5.2$ & $<0.001$ \\
\hline TMT-B (second) & $210.9 \pm 70.1^{\star \star \star}$ & $197.1 \pm 72.1$ & $187.3 \pm 74.1$ & $<0.001$ \\
\hline \multicolumn{5}{|c|}{$\begin{array}{l}\text { Data was represented as mean } \pm \text { SD. Kruskal Wallis tests followed by Dunn-Bonferroni post hoc were } \\
\text { performed as the neuropsychological data were not normally distributed }(P<0.05 \text { for Kolmogorov- } \\
\text { Smirnov test). The } \chi 2 \text { tests were performed for gender. *Indicates a statistical difference compared } \\
\text { with HC. * indicate } P<0.05, \text { ** indicate } P<0.01 \text { and } * \star * \text { indicate } P<0.001 \text {. MMSE: Mini-Mental State } \\
\text { examination; AVLT-20, Auditory Verbal Learning Test-20-minute delayed recall; CFT20, Rey-Osterrieth } \\
\text { Complex Figure Test-20-minute delayed recall; CDT, Clock Drawing Test; DSST, Digital Symbol } \\
\text { Substitution Test; TMT-A, Trail Making Test-A; VFT, Verbal Fluency Test; TMT-B, Trail Making Test-B. }\end{array}$} \\
\hline
\end{tabular}

\subsection{Identification of disease-related differences in DMN}

As illustrated by the one-sample t-test, the DMN patterns for each group were obtained by using PCC seed-based FC analysis (seen in Fig. 1).

As shown by one-way ANOVA, the disease-related differences in DMN were detected in the bilateral precuneus (BPcu) (details see Table 2 and Fig. 2). It should be noted that the region mentioned above was taken as ROI. 
Table 2

Descriptions of disease-related differences in DMN revealed by one-way ANOVA

\begin{tabular}{|c|c|c|c|}
\hline Brain region & $\begin{array}{l}\text { Peak MNI } \\
\text { Coordiates } \\
x, y, z(m m)\end{array}$ & $\begin{array}{l}\text { Peak } \\
\text { F value }\end{array}$ & $\begin{array}{l}\text { Cluster size } \\
\left(\mathrm{mm}^{3}\right)\end{array}$ \\
\hline Bilateral Precuneus & $-9,-63,51$ & 12.4 & 6615 \\
\hline
\end{tabular}

\subsection{Group-level differences and behavioral significance of the genotype-by-disease interaction in DMN}

Among the three groups, significant FC differences were detected within the BPcu $(F=10.7, P<0.001)$. The LSD analysis indicated that compared to PD-NC and PD-MCl, the HCs showed significantly increased FC within BPcu (both $P<0.001$ ). Further, there was also significant difference regarding the FC within BPcu between the two PD groups ( $P<0.001$, details seen in Fig. 3$)$.

As illustrated in Fig. 4, for PD-MCl, the higher the FC values within BPcu were related to the better the performances information processing speed (i.e. shorter time consumed of TMTA, rho $=-0.50, P=0.033$, and higher scores of DSST, rho $=-0.66, P=0.003$ ) and episodic memory (i.e. higher scores of CFT-20 min $\mathrm{DR}$, rho $=0.47, P=0.047)$. Similar associations were also detected between the FC within BPcu and performances of MMSE (rho $=0.58, P=0.046)$ for PD-NC.

\section{Discussion}

The primary objective of this study was to explore the difference of pattens of anatomical and FC in $\mathrm{HCs}$, $\mathrm{PD}$, and $\mathrm{PD}-\mathrm{MCl}$ and the relationship between the connection strengths and cognitive statelWe found that in PD patients, brain network associated with the cognitive dysfunction was BPcu, and the FC within BPcu significantly decreased in PD-NC and PD-MCl. Furthermore, higher connectivity strength was associated with a faster information processing speed (TMTA, DSST), a better episodic memory (CFT20 min DR) and a better general cognition (MMSE).

Rs-fMRI is a promising approach that measures naturally occurring low-frequency fluctuations in blood oxygenation level-dependent (BOLD) signals reflecting physiologically meaningful changes of spontaneous neural activity in the resting-state networks (RSNs) to investigate neuropsychiatric disorders[15]. It avoids demanding task performance and can easily obtain relatively stable results[16]. Rs-fMRI is widely used in Alzheimer's disease (AD)[17], other dementias[18] and mood disorders[15]. Mounting studies have identified a wide array of brain regions that exhibit group-wise differences between HCs and neuropsychiatric disorders[15]. Over the last decades, rs-fMRI has been carried out to 
investigate the FC changes in patients with PD [19-22]. However, there is still a need for understanding the exact relationship between the function and brain regions and the relationship between the connection strengths and cognitive state.

DMN is the most popular target in RSNs, including a midline core (including the anterior medial prefrontal cortex (aMPFC) and PCC) and two subsystems (the dorsal medial prefrontal cortex (DMPFC) subsystem and the medial temporal lobe (MTL) subsystem )[23]. DMN is thought to be involved in advanced cognitive functions. Early study has observed DMN dysfunction in PD patients[24]. Wolters et al. [25] observed that cognitive impairment in PD was correlated with reduced FC in networks involved in cognition, especially in the DMN. Studies suggested that the precuneus was implicated in high-level cognitive functions, including self-related processing, episodic memory, and aspects of consciousness [26-28]. Our study found that BPcu was the cognitive related region in DMN.

There is significant clinical heterogeneity in cognitive impairment in $\mathrm{PD}[29]$. The prevalence of PD-MCI was $25 \%[29]$ and with the development of the disease, the cumulative prevalence of PD with dementia (PDD) gradually increases[18]. The frequency and severity of PD induced cognitive decline emphasise the need to approach this impairment as a symptom that requires separate attention. However, the mechanism of PDD is not clear yet. It is urgently need to find biomarkers or identify the risk factors or signal of PDD in early stage for improving the prognosis of the disease[30]. The PD-NC and HCs in our study showed comparable performances of cognitive function, but the FC within the cognitive-related region in the brain - BPcu in PD-NC was significantly declined compared to HCs, which might be an early signal of cognitive damage. Díez-Cirarda et al. [19] included $26 \mathrm{HCs}, 12 \mathrm{PD}-\mathrm{NC}$ and $23 \mathrm{PD}-\mathrm{MCl}$ and found that PD-MCl group dynamic FC deteriorated but did not been observed in PD-NC.

The cognitive impairment of PD patients is mainly manifested in visual space, attention, executive function, and memory, but these clinical manifestations are not diagnostic specific[5]. Similar with the report, $\mathrm{PD}-\mathrm{MCl}$ in our study showed significant worse performances in general cognition. For PD-MCl, the higher the FC values within BPcu were related to better performances information processing speed (i.e. shorter time consumed of TMTA and higher scores of DSST) and episodic memory (i.e. higher scores of CFT-20 min DR). Similar associations were also detected between the FC within BPcu and performances of MMSE for PD-NC.

There are some limitations in this study. First, this is a single center study with a limited number of patients. Also, all PD patients from this study were on medication and we cannot rule out the possibility that the drugs may have impacted the rs-fMRI. Lastly, the use of the outcomes needs to be further validated in additional populations.

\section{Conclusions}

In conclusion, BPcu was the cognitive related region in DMN. As cognition declines, FC within BPcu weaken. For PD-MCl, the higher the FC values within BPcu were related to the better the performances of 
TMTA, DSST and CFT-20 min DR. For PD patients with normal cognition, the FC within BPcu were positively correlated with scores of MMSE.

\section{Abbreviations}

PD:Parkinson's disease; rs-fMRI:resting state functional magnetic resonance imaging; FC:functional connectivity; PD-MCl:PD patients with mild cognitive impairment; PD-NC:PD patients with normal cognition; HCs:healthy controls; MMSE:Mini-Mental State Examination; AVLT-20 min DR:Auditory Verbal Learning Test-20-min delayed recall; CFT-20 min DR:Rey-Osterrieth Complex Figure Test; TMT:Trail Making Test; VFT:Verbal Fluency Test; DSST:Digital Symbol Substitution Test; CDT:Clock Drawing Test; CDR:Clinical Dementia Rating; NINCDS-ADRDA:National Institute of Neurological and Communicative Disorders and Stroke and the Alzheimer's Disease and Related Disorders Association; TR:repetition time; TE:echo time; FA:flip angle; FOV:field of view; NEX:no. of excitations; PCC:posterior cingulate cortex; CC:cross-correlation; DMN:default mode network; VBM:voxel-based morphometry; ANOVA:One-way analysis of variance; zFC:Z-transformed FC; LSD:least-significant difference; BPcu:bilateral precuneus; BOLD:blood oxygenation level-dependent; RSNs:resting-state networks; aMPFC:anterior medial prefrontal cortex; DMPFC:dorsal medial prefrontal cortex; MTL:medial temporal lobe; PDD:PD with dementia.

\section{Declarations}

Ethics approval and consent to participate

The study was approved by the medical ethics committee of the Affiliated Jiangyin People's Hospital of Southeast University Medical College. Written consent was obtained from all participants

Consent to publish

Not applicable.

Availability of data and materials

The datasets analysed during the current study are available from the corresponding author on reasonable request.

Competing interests

None of the authors report a conflict of interest.

\section{Funding}

This work was funded by the Guidance Plan for Social Development of Taizhou Municipal Science and Technology (ssf20160141). The funder had no role in the study design, data collection, data analysis, data interpretation, writing of the report, decision to publish, or preparation of the manuscript.

Authors' contributions

Study concept and design: JLN, YLD, QGW and WH. Data analysis: QGW and WH. Data collection: QGW, WH, DHL, BJH, QTJ and YLD. Writing of the manuscript: QGW, WH and YLD. Revising the manuscript: 
JLN, BJH and QTJ. Final approval of the manuscript: all.

Acknowledgements

Not applicable.

\section{References}

1. Grimes D, Fitzpatrick M, Gordon J, Miyasaki J, Fon EA, Schlossmacher M, Suchowersky O, Rajput A, Lafontaine AL, Mestre T, et al. Canadian guideline for Parkinson disease. Cmaj. 2019;191(36):E9891004.

2. Zhang ZX, Roman GC, Hong Z, Wu CB, Qu QM, Huang JB, Zhou B, Geng ZP, Wu JX, Wen HB, et al. Parkinson's disease in China: prevalence in Beijing, Xian, and Shanghai. Lancet. 2005;365(9459):595-7.

3. Ma CL, Su L, Xie JJ, Long JX, Wu P, Gu L: The prevalence and incidence of Parkinson's disease in China: a systematic review and meta-analysis. Journal of neural transmission (Vienna, Austria: 1996) 2014, 121(2):123 - 34.

4. Litvan I, Bhatia KP, Burn DJ, Goetz CG, Lang AE, McKeith I, Quinn N, Sethi KD, Shults C, Wenning GK. Movement Disorders Society Scientific Issues Committee report: SIC Task Force appraisal of clinical diagnostic criteria for Parkinsonian disorders. Movement disorders: official journal of the Movement Disorder Society. 2003;18(5):467-86.

5. Boyu Chen. Multi-modal MRI study of cognitive dysfunction in Parkinson's disease. PhD. China Medical University; 2017.

6. Chinese Medical Association Neurology Branch Parkinson's Disease and Movement Disorders Group;Chinese Medical Doctor. Association of Neurology,Parkinson's Disease and Movement Disorders Committee: Chinese diagnostic criteria for Parkinson's disease[J]. Chin J Neurol. 2015;48(11):1015-9.

7. Petersen RC, Smith GE, Waring SC, Ivnik RJ, Tangalos EG, Kokmen E. Mild cognitive impairment: clinical characterization and outcome. Arch Neurol. 1999;56(3):303-8.

8. Albert MS, DeKosky ST, Dickson D, Dubois B, Feldman HH, Fox NC, Gamst A, Holtzman DM, Jagust WJ, Petersen RC, et al. The diagnosis of mild cognitive impairment due to Alzheimer's disease: recommendations from the National Institute on Aging-Alzheimer's Association workgroups on diagnostic guidelines for Alzheimer's disease. Alzheimer's dementia: the journal of the Alzheimer's Association. 2011;7(3):270-9.

9. McKhann GM, Knopman DS, Chertkow H, Hyman BT, Jack CR Jr, Kawas CH, Klunk WE, Koroshetz WJ, Manly JJ, Mayeux R, et al. The diagnosis of dementia due to Alzheimer's disease: recommendations from the National Institute on Aging-Alzheimer's Association workgroups on diagnostic guidelines for Alzheimer's disease. Alzheimer's dementia: the journal of the Alzheimer's Association. 2011;7(3):263-9. 
10. Buckner RL, Sepulcre J, Talukdar T, Krienen FM, Liu H, Hedden T, Andrews-Hanna JR, Sperling RA, Johnson KA. Cortical hubs revealed by intrinsic functional connectivity: mapping, assessment of stability, and relation to Alzheimer's disease. The Journal of neuroscience: the official journal of the Society for Neuroscience. 2009;29(6):1860-73.

11. Shu H, Shi Y, Chen G, Wang Z, Liu D, Yue C, Ward BD, Li W, Xu Z, Chen G, et al. Opposite Neural Trajectories of Apolipoprotein E 4 and 2 Alleles with Aging Associated with Different Risks of Alzheimer's Disease. Cerebral cortex. 2016;26(4):1421-9.

12. Bai F, Liao W, Yue C, Pu M, Shi Y, Yu H, Yuan Y, Geng L, Zhang Z. Genetics pathway-based imaging approaches in Chinese Han population with Alzheimer's disease risk. Brain structure function. 2016;221(1):433-46.

13. Inkster B, Nichols TE, Saemann PG, Auer DP, Holsboer F, Muglia P, Matthews PM. Association of GSK3beta polymorphisms with brain structural changes in major depressive disorder. Arch Gen Psychiatry. 2009;66(7):721-8.

14. Inkster B, Nichols TE, Saemann PG, Auer DP, Holsboer F, Muglia P, Matthews PM. Pathway-based approaches to imaging genetics association studies: Wnt signaling, GSK3beta substrates and major depression. Neurolmage. 2010;53(3):908-17.

15. Takamura T, Hanakawa T: Clinical utility of resting-state functional connectivity magnetic resonance imaging for mood and cognitive disorders. Journal of neural transmission (Vienna, Austria: 1996) 2017, 124(7):821 - 39.

16. Pan $P$, Zhang $Y$, Liu Y, Zhang $H$, Guan $D$, Xu Y. Abnormalities of regional brain function in Parkinson's disease: a meta-analysis of resting state functional magnetic resonance imaging studies. Scientific reports. 2017;7:40469.

17. Greicius MD, Srivastava G, Reiss AL, Menon V. Default-mode network activity distinguishes Alzheimer's disease from healthy aging: evidence from functional MRI. Proc Natl Acad Sci USA. 2004;101(13):4637-42.

18. Bai F, Zhang Z, Yu H, Shi Y, Yuan Y, Zhu W, Zhang X, Qian Y. Default-mode network activity distinguishes amnestic type mild cognitive impairment from healthy aging: a combined structural and resting-state functional MRI study. Neurosci Lett. 2008;438(1):111-15.

19. Diez-Cirarda M, Strafella AP, Kim J, Pena J, Ojeda N, Cabrera-Zubizarreta A, Ibarretxe-Bilbao N. Dynamic functional connectivity in Parkinson's disease patients with mild cognitive impairment and normal cognition. Neurolmage Clinical. 2018;17:847-55.

20. Prajapati R, Emerson IA. Global and regional connectivity analysis of resting-state function MRI brain images using graph theory in Parkinson's disease. The International journal of neuroscience 2020:111.

21. Lee YH, Bak Y, Park CH, Chung SJ, Yoo HS, Baik K, Jung JH, Sohn YH, Shin NY, Lee PH. Patterns of olfactory functional networks in Parkinson's disease dementia and Alzheimer's dementia. Neurobiology of aging 2019. 
22. Chung SJ, Kim HR, Jung JH, Lee PH, Jeong Y, Sohn YH. Identifying the Functional Brain Network of Motor Reserve in Early Parkinson's Disease. Movement disorders: official journal of the Movement Disorder Society 2020.

23. Hou Y, Yuan X, Wei Q, Ou R, Yang J, Gong Q, Shang H: Primary disruption of the default mode network subsystems in drug-naive Parkinson's disease with mild cognitive impairments. Neuroradiology 2020.

24. van Eimeren T, Monchi O, Ballanger B, Strafella AP. Dysfunction of the default mode network in Parkinson disease: a functional magnetic resonance imaging study. Arch Neurol. 2009;66(7):87783.

25. Wolters AF, van de Weijer SCF, Leentjens AFG, Duits AA, Jacobs HIL, Kuijf ML. Resting-state fMRI in Parkinson's disease patients with cognitive impairment: A meta-analysis. Parkinsonism Relat Disord. 2019;62:16-27.

26. Cavanna AE, Trimble MR. The precuneus: a review of its functional anatomy and behavioural correlates. Brain. 2006;129(Pt 3):564-83.

27. Vogt BA, Laureys S. Posterior cingulate, precuneal and retrosplenial cortices: cytology and components of the neural network correlates of consciousness. Prog Brain Res. 2005;150:205-17.

28. Cavanna AE. The precuneus and consciousness. CNS Spectr. 2007;12(7):545-52.

29. Margulies DS, Böttger J, Long X, Lv Y, Kelly C, Schäfer A, Goldhahn D, Abbushi A, Milham MP, Lohmann $G$, et al. Resting developments: a review of $f M R I$ post-processing methodologies for spontaneous brain activity. Magma (New York NY). 2010;23(5-6):289-307.

30. Chinese Medical Association Neurology Branch Parkinson's Disease and Movement Disorders Group;Chinese Medical Doctor. Association of Neurology,Parkinson's Disease and Movement Disorders Committee: Chinese expert consensus on research criteria for prodromal Parkinson's disease. Chinese Journal of Geriatrics. 2019;38(8):825-31.

\section{Figures}




\section{HC}
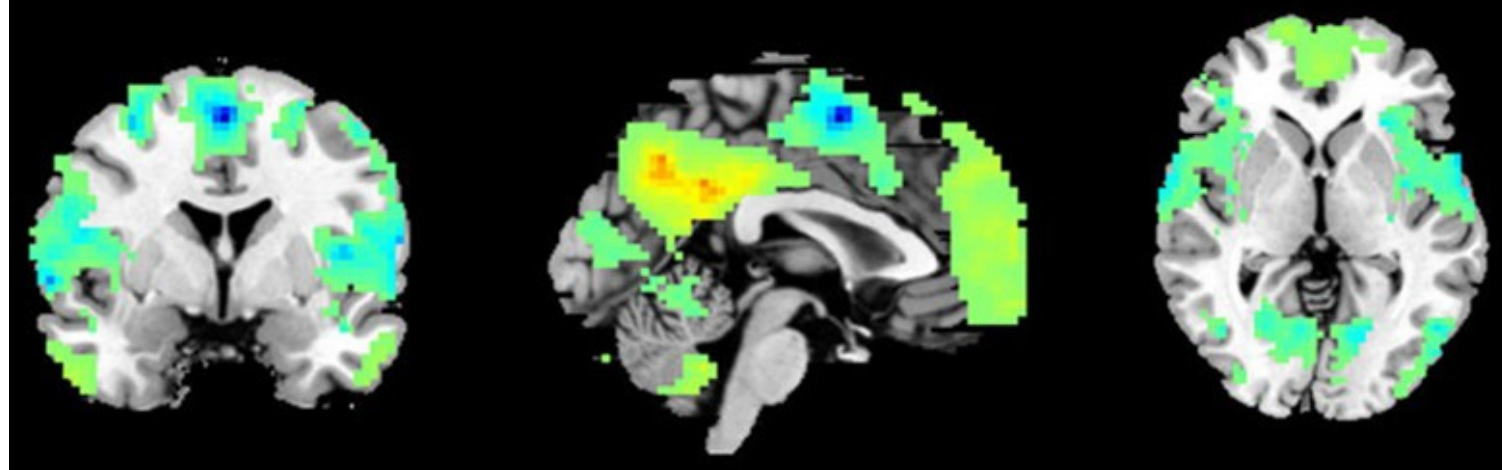

\section{PD with normal cognition}
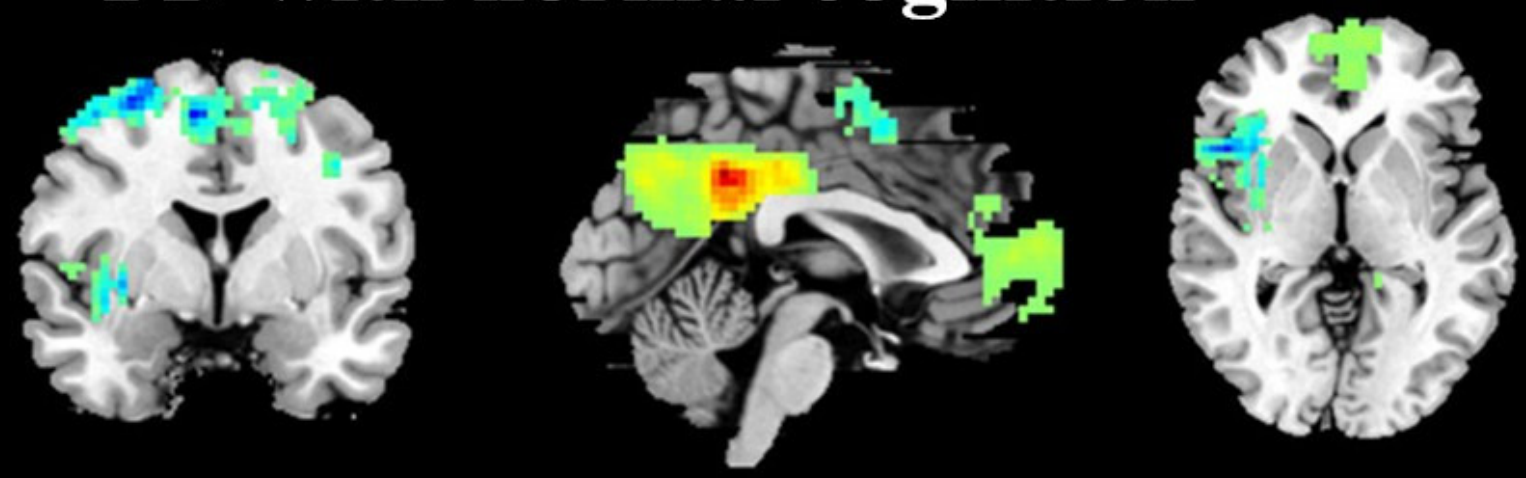

\section{PD with MCI}
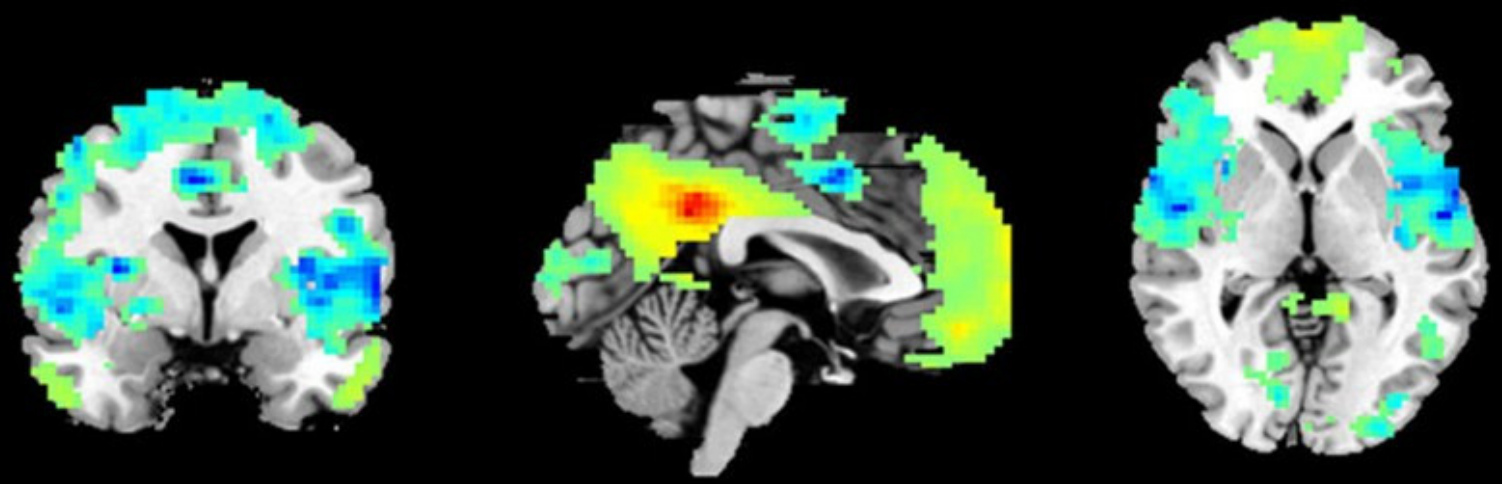

Figure 1

Whole-brain voxelwise pattern of the default mode network in each group. The DMN pattern for each group was obtained by using PCC (MNI coordinate: $-2,-45,34$, radius $=6 \mathrm{~mm}$ ) seed-based functional activity analysis. The random-effects one-sample t-test was performed and threshold was set at a corrected $\mathrm{P}<0.01$, determined by Monte Carlo simulation. 

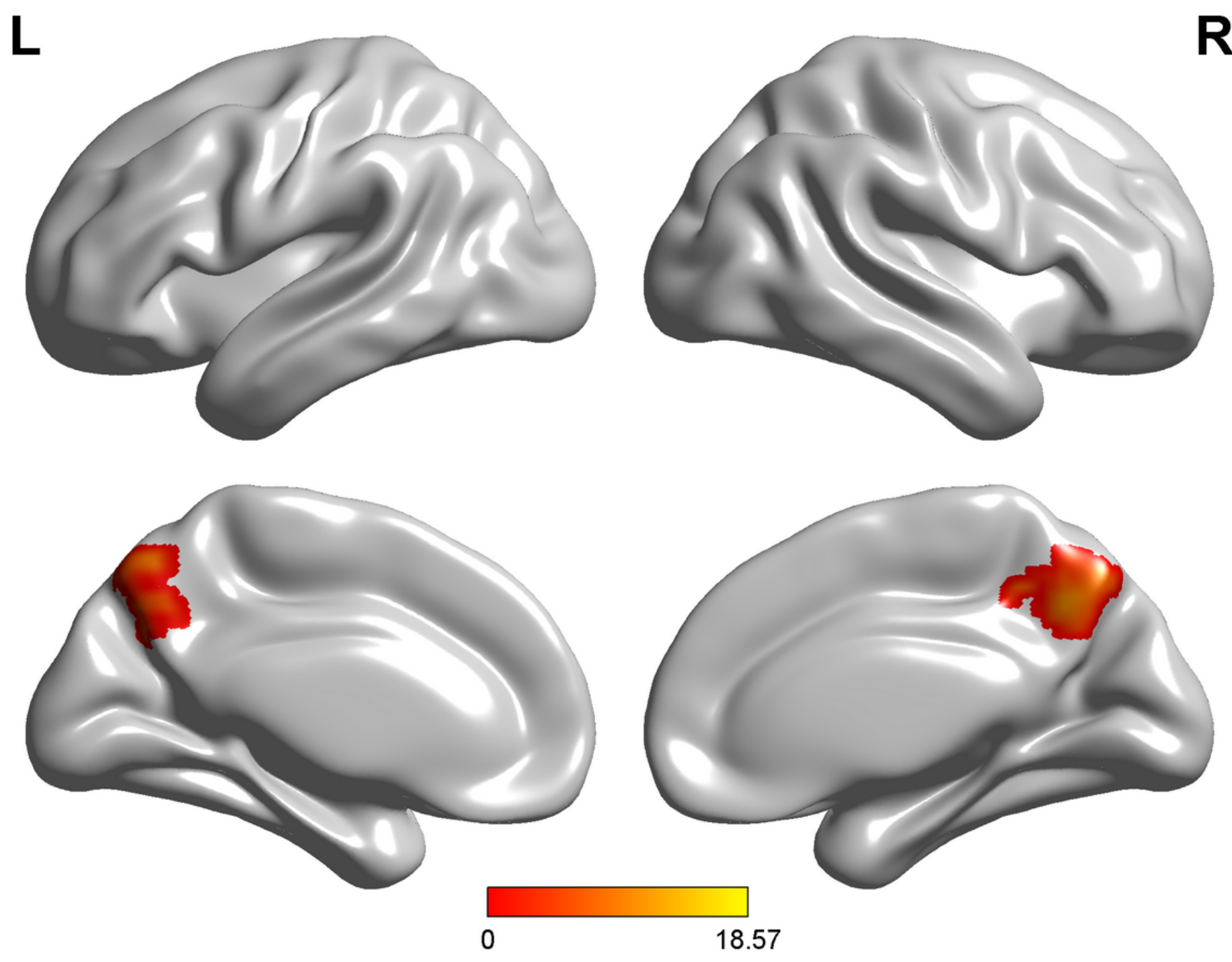

Figure 2

One-way ANOVA of DMN functional connectivity. Thresholds were set at a corrected $\mathrm{P}<0.01$, determined by Monte Carlo simulation. 


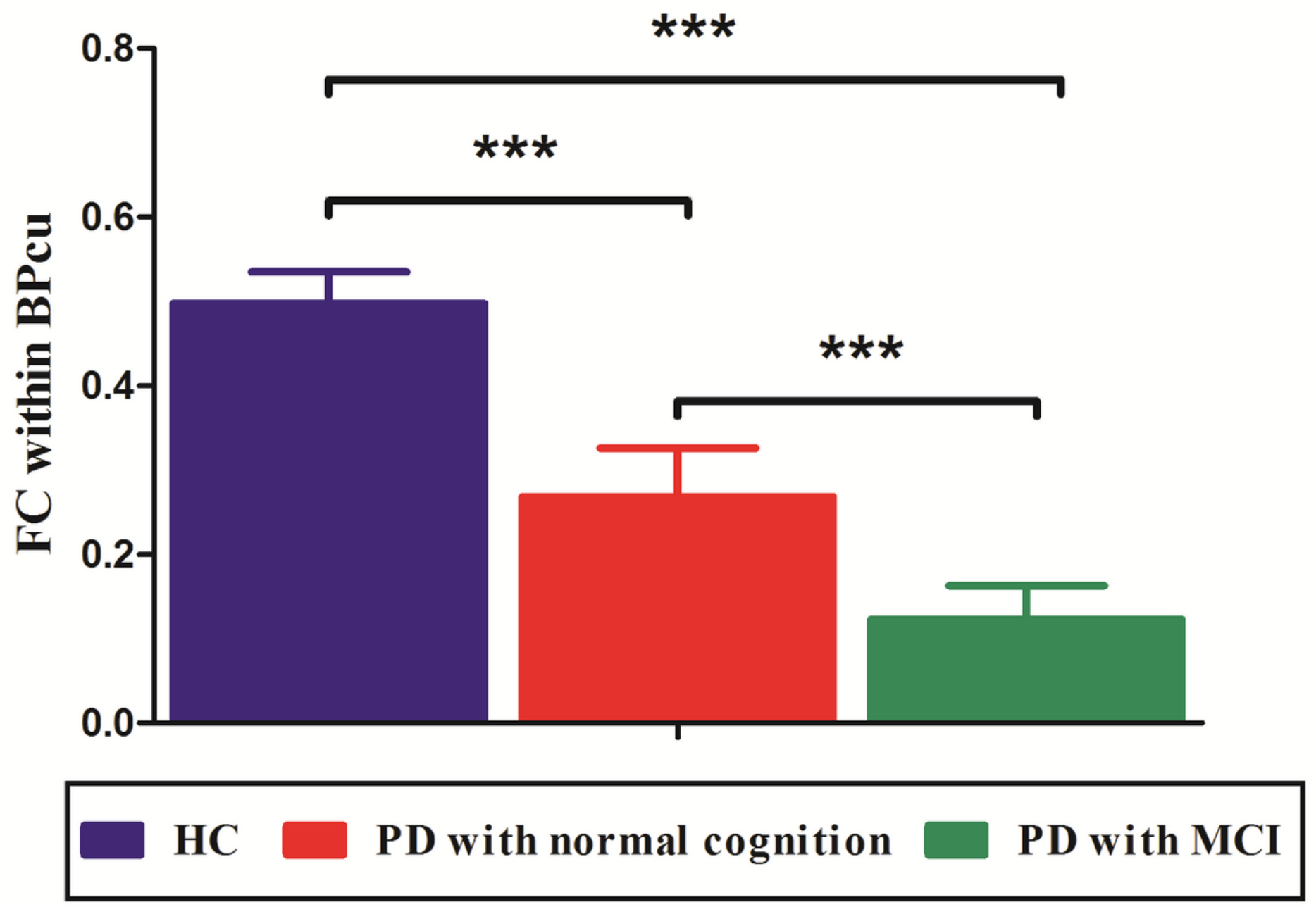

Figure 3

Group-level differences of the disease-related differences in DMN. Compared to PD patients with normal cognition and PD patients with $\mathrm{MCl}$, the HCs showed significantly increased $\mathrm{FC}$ within BPcu. Further, there was also significant difference regarding the FC within BPcu between the two PD groups. *ᄎ* indicate $\mathrm{P}<$ 0.001 . 


\section{PD with MCI}

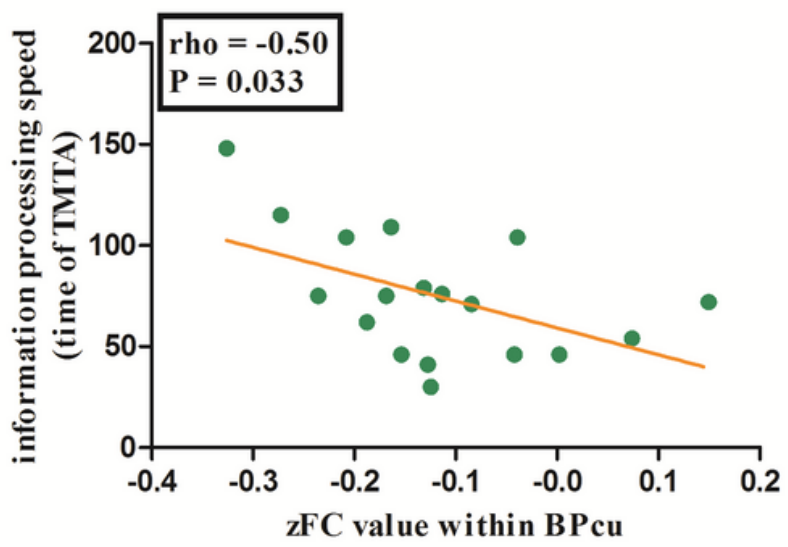

PD with MCI

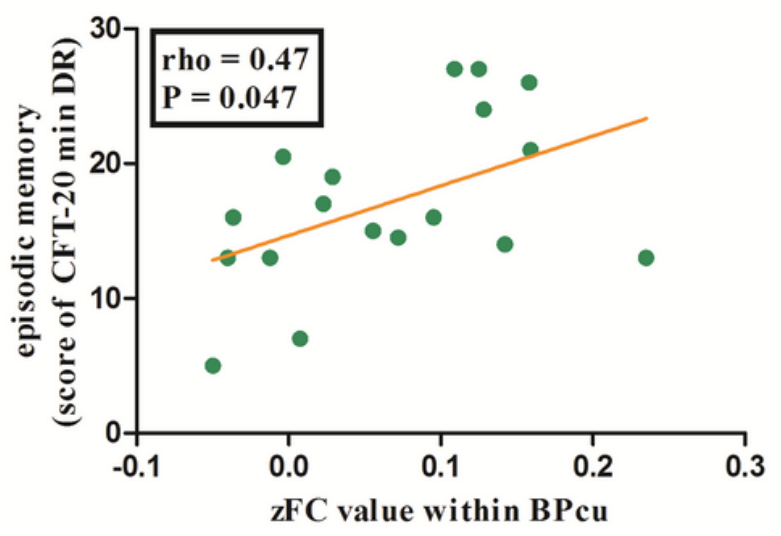

PD with MCI
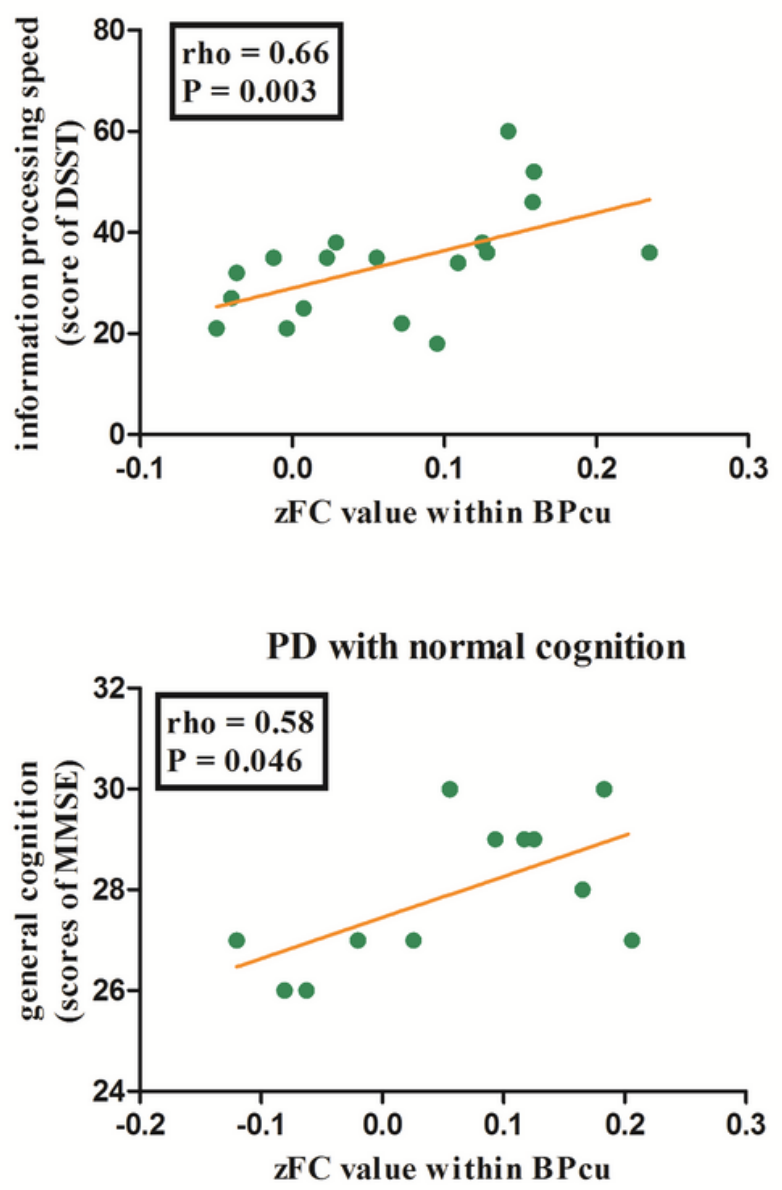

\section{Figure 4}

Behavior significances of the regions with disease-related differences in DMN. For PD patients with $\mathrm{MCl}$, the higher FC within BPcu were related to better performances of TMTA, DSST and CFT-20 min DR. For PD patients with normal cognition, the FC within BPcu were associated with scores of MMSE. 